\title{
Is rudeness really that common? An exploratory study of incivility at work
}

\author{
Kim A. McCarthy \\ Management Department, College of Business Administration, California State University, San Marcos, California, USA
}

\begin{abstract}
Currently, most studies of incivility involve surveys and controlled laboratory experiments that focus on examining the associations between incivility and other variables. This method of investigation is important, but insufficient for developing a comprehensive understanding of such a complex and multifaceted construct. The present study explores employees' perceptions of rude workplace behavior from the perspective of real employees in managerial roles. It investigates the extent to which incivility is a prevalent issue faced by employees at work, examines the perceived impact of incivility on relational and productivity-related outcomes, and, finally, considers the potential differences between face-toface rudeness and electronic rudeness. A qualitative interview approach is used to explore and analyze employees' perceptions and investigate what employees think, feel, and say about their experiences with incivility at work.
\end{abstract}

\section{KEYWORDS}

Computer-mediated communication; cyber incivility; incivility; rudeness; workplace communication

\section{Introduction}

"Business people are supposed to be rude," was what a participant wrote in the margin of a survey developed to better understand workplace incivility. The respondent had crossed out the possible choices and written this statement. After talking with employees in a wide range of occupations about their experiences with rudeness, it became clear that the meaning and reason for incivility is as nuanced and complicated as the people involved. Therefore, instead of relying on a survey to better understand it, a more flexible approach was necessary to adequately learn about employees' experiences and explore their perspectives without trying to quantify and categorize their responses. The respondent's comment was an indication that the quantitative methods, which currently dominate research on incivility, are lacking in their ability to capture the subtleties of the phenomenon. This study begins to bridge that gap by complementing the many existing quantitative studies, with a qualitative study aimed at gaining a better understanding of incivility from the perspective of real employees who experience it.

In the management literature, rudeness is defined as workplace behavior perceived to be insensitive or disrespectful (Lim and Teo 2009; Pearson, Andersson, and Wegner 2001). It is a low-intensity antisocial behavior which lacks a clear intent to harm (Andersson and Pearson 1999), in that it may or may not be intentional, but nonetheless violates social norms and is perceived as rude by the target (Cortina 2008). Rudeness, which can also be referred to as incivility (Porath and Erez 2007), can compromise the well-being of organizations as well as the commitment of its members, and carries with it adverse social, attitudinal, and behavioral consequences (Giacalone, Riordan, and Rosenfeld 1997; Griffin, O’Leary-Kelly, and Collins 1998; O’Leary-Kelly, Griffin, and Glew 1996). Despite the serious- ness of its impact, and growing interest in the topic, research up to this point has mostly focused on understanding it through surveys and laboratory studies (Miron-Spektor et al. 2011; Porath and Pearson 2010; Taylor, Bedeian, and Kluemper 2012), which arguably, provides an important, albeit incomplete, baseline for understanding the subtleties of employees' encounters with rude behavior at work.

In the management literature, research is currently more focused on examining its associations with outcomes, 
such as individual performance (Porath and Erez 2007), employee well-being (Cortina et al. 2001), affective commitment (Minor et al. 2012), turnover (Johnson and Indvik 2001), and satisfaction (Pearson and Porath 2005). Although these studies and developments are undoubtedly important and help highlight the adverse effects of incivility, quantitative research by itself is limited in terms of its capacity to explore the nuances and complexities of a multifaceted phenomenon. Instead, the central question this research addresses is how employees' experiences with incivility at work inform what is already known about rude behavior. Specifically, three questions are examined in this research, each one of which is explained and discussed in the following sections. Then, a qualitative interview approach, similar to the one used by Penconek (2015), is employed to investigate these questions about rudeness. Finally, findings are reported, their implications are discussed, and questions for future research are proposed.

\section{Background and theoretical motivation}

There are three primary questions that motivate this work and the examination of incivility from the perspective of real employees who experience it: (1) the extent to which incivility is an issue at work, including its perceived impact on relationships, attitudes, and work quality; (2) the context and situational factors that contribute to its occurrence and severity; and (3) the potential differences between employees' experience of rudeness through electronic versus face-to-face mediums. Each question is addressed individually in the following sections.

\subsection{Incivility as a workplace issue}

The first benefit of exploring incivility from the perspective of real employees, is to examine the frequency with which employees' experience it and the extent to which it poses a real threat to organizations. In other words, how often do employees endure rude behavior? Is it encountered daily? Weekly? Or even on a monthly basis? It is possible that employees experience rude treatment much less frequently than previously believed. Specifically, given that it is a low intensity behavior, its presumed impact has been called into question (Hershcovis 2011) compared to other more severe forms of workplace aggression. In fact, its status as a low intensity behavior (Andersson and Pearson 1999) makes the question of frequency even more significant. For instance, a mild aggravation that occurs infrequently may very well have a limited impact. On the other hand, research indicates that a continuous minor stressor may pose a greater threat to employees than a more major life crisis (Cortina 2008; Cortina et al. 2001; Deitch et al. 2003; Ivancevich 1986).

The question of whether incivility poses a threat to employees and organizations is important, but not new. Several existing studies have addressed the frequency with which employees encounter incivility at work (Giumetti et al. 2013; Jessmer and Anderson 2001; Pearson and Porath 2005; Taylor, Bedeian, and Kluemper 2012; Turnage 2008). However, these studies overwhelmingly involve surveys, which ask participants to rate the frequency that they experience incivility on prescribed intervals of time (Pearson and Porath 2005) or to choose the regularity with which they experience a predetermined list of rude behaviors (Lim and Teo 2009). Unfortunately, for a phenomenon such as incivility, using a survey is limiting and highly susceptible to researcher bias.

\subsection{Context and situational factors}

A second reason for exploring incivility using a qualitative approach is to investigate the contextual aspects and circumstances that accompany instances of incivility from the perspective of employees. Several current studies on incivility have focused on examining the outcomes associated with rude behavior (i.e., Cameron and Webster 2011; Giumetti et al. 2013; Lim and Teo 2009; Porath and Erez 2007) as well as the factors that lead to it (Francis, Holmvall, and O'Brien 2015), and yet most have neglected to pay adequate attention to the contextual features surrounding instances of rudeness. Some scholars have even called specifically for more research on the 
antecedents of rudeness (Weatherbee 2010), while at the same time, identifying the challenges associated with studying the context of such a complicated and multidimensional phenomenon. The present study includes in-depth questions about who was involved, where the situation took place, and how it developed and progressed. By talking to employees about their real-life experiences with rude behavior, it is possible to probe deeper when necessary and ultimately understand the situation in a more complete and dynamic way.

\subsection{Rude email versus face-to-face}

Another pertinent question is whether employees' experiences with rudeness through electronic modes such as email, social media, and text messaging differ from their experiences with face-to-face rudeness. Prior research has introduced some compelling reasons to consider making a distinction between face-to-face and electronic forms of rudeness (Derks, Bos, and Grumbkow 2007; Naquin, Belkin, and Kurtzberg 2010). For instance, the distinct contextual features of electronic messaging are likely to affect rudeness perceptions (Riordan and Kreuz 2010). Electronic communication is most often text-based, asynchronous, lacking in accompanying paralinguistic signals (facial expressions, hand gestures, and so forth), and back-channeling cues (e.g., head-nodding), all of which are likely to affect employees' perceptions of rudeness (Burgoon and Buller 1994). In the case of electronic incivility, a message receiver is left to interpret messages without the help of these contextual features, making it more likely that a message will be miscommunicated and interpreted as rude (Kato, Kato, and Scott 2009). These reasons would support making a distinction between face-to-face and electronic forms of rudeness.

On the other hand, some scholars argue that the differences between face-to-face and electronic forms of rudeness are less significant (Walther, Anderson, and Park 1994), and splitting the constructs only leads to unnecessary proliferation (Derks, Fischer, and Bos 2008; Hershcovis 2011). Therefore, an important question this research examines is whether employees themselves perceive differences between forms of incivility. For instance, do employees talk about them using different terms? Do they cope with them differently? Do instances of each seem to have the same outcomes and consequences? Are there differences in terms of the prevalence of rudeness through each form? By asking employees to explain their experiences, it will be possible to learn more about this important question.

\section{Study design and methodology}

To test the questions posed, the qualitative study was comprised of a snowball sample of 15 organizational professionals. Only employees who held managerial positions in white-collar jobs, across a wide-range of industries and occupations, and spent time using email as a means of communication at work on a daily basis were included in the interview sample set. The goal was to select individuals who were representative of a broad range of professional occupations and managerial-level careers in a number of different fields. Participants represented industries such as healthcare, education, oil and gas, engineering, entertainment, and e-marketing. All participants had managerial experience, and were in positions requiring the daily use of email to communicate, coordinate, and direct other employees and clients.

Data collection consisted of semi-structured interviews, which lasted approximately one hour to one and a half hours each. Each interview started with a series of basic questions about the participant's email use at work. These included questions about participants' experiences with incivility, the approximate number of instances of rudeness they remember, and with whom those situations took place. These questions focused on understanding the norms of conduct in their organization. For instance, questions were asked such as, "About how often is someone at work rude to you?" Also, "Is it usually the same person, or is it various different people? Please tell me more about these situations."

The next portion of the interview focused on uncovering how employees identified, classified, thought and felt about, as well as responded to, rude workplace behavior. Thus, the questions included, "How do you handle workplace behavior that you consider to be rude?", "What are some of the outcomes?", "Has it affected your 
working relationships or the working relationships of your co-workers?", "Specifically, what do you think leads to this type of behavior?", and "How is it perpetuated?" Finally, to explore the affective, cognitive, and behavioral consequences of receiving a rude email, interviewees were asked to "...recall a time when they have been the victim of rudeness....", "How did it make you feel?", "What did you think?", "About how much time did you spend thinking about it?", "Did you worry about it?", and "How did you decide to respond?"

To determine whether people perceived a difference between rude email and rude face-to-face encounters, they were asked "Do you think receiving a rude email is different from someone acting rudely to you in person at work?", "Which scenario do you think happens more often?", "Is one usually worse than the other?", "Why? Or Why not?", "Is one more likely to be resolved in a positive way?", "Why?", and "Can you give me an example?" Although the interview followed a script, the interviewees were also asked other probing questions relevant to examples and stories they shared.

In general, the interview questions were devised to investigate specific aspects, features, and instances of incivility. They were designed to produce an interview dataset that consisted of both the participant's answers to direct questions about rudeness, as well as capture answers in response to indirect questions about the topic. The latter set of responses reveal additional features of the construct, which participants did not include when asked about the phenomenon directly. Following the completion of data collection, participants' responses were transcribed to form a comprehensive qualitative database. The data from approximately 15 hours of interviews were transcribed into one qualitative archive for the exploration of employee perceptions of incivility using interview data.

\section{Analysis and results}

The interview data furnishes evidence that employees do encounter rude behavior regularly in organizations, they have opinions about what leads to incivility, and employees tend to believe it has adverse consequences for themselves, their workplace relationships, and their productivity. As suggested by the following interview excerpts, the data indicate initial support for the notion that rudeness in either form is likely quite prevalent in organizations:

Although I don't get as much now in my current job, when I was working for (name of company) a few years ago, it was a very high stress environment, and I would say I got a rude email probably on a monthly basis... (Consultant, Public Relations)

In our industry it's probably a little different, because it just depends on the job, and sometimes you can end up working with a contractor that's not very nice. ... I would say I get treated rudely about once a month. (Structural Engineer, Construction Engineering)

"It happens so many times in my line of work where someone will send an email and it's like they need something, you know, "right now" ... and that can really make people mad.

(Title Analyst, Oil and Gas)

One observation noticed early-on in the process of conducting the interviews was that participants seemed to be more comfortable recalling instances of rudeness that occurred in the past or when the rudeness was directed toward another coworker instead of toward themselves. Commonly, interviewees would say that "things others think are rude don't bother them" or they "let most things roll off their back." However, as the interview progressed, and the topic was approached in a different way, the same interviewees seemed more forthcoming with examples of rudeness.

This may have been because they simply could not recall any instances of rudeness when asked about it initially, and it took some thinking before they could remember specific examples. In support of this reasoning, instances of rudeness are characteristically "low intensity" acts; therefore, examples would most likely be less memorable than other more intense acts of workplace aggression. However, this may also be evidence that admitting to being offended or hurt by incivility at work is a sensitive topic and one that people feel more 
comfortable discussing at a psychological distance, (i.e., far in the past, something experienced only by coworkers) or only with close trusted friends. As the following excerpts suggest, when faced with recalling examples of rudeness, some interviewees gave an initial response that was very non-committal, but then disclosed more information later in the interview:

Uuuuum. I'd say it's not that common. We tend to have really good relationships with our clients, so there is an understanding $\ldots$ and we know they're busy.

(after question was rephrased)

The rudeness tends to come from clients not responding to us at all ... or one of the things that especially bothers me and a lot of my co-workers, is when someone ... will write an email with multiple parts or multiple questions, and they (the client) obviously hasn't read it and they'll just reply back with some sort of a question that was answered at a point later in the email ...

(Senior Account Manager, e-Marketing Firm)

The next example is an interviewee who was initially quite certain that he had never been the victim workplace incivility, but after asking more specific questions and focusing on describing a particular scenario from the past, he became more forthcoming:

I think I probably have a fairly high tolerance for that kind of thing, you know what I mean? It's like if you look at it in terms of a gray area, you'd probably have to come right out and be nasty to me before I would consider it rude.

(after interviewee was asked more specific questions about an incident)

Most of the rudeness I have experienced has been from the same person. This coworker just has more of a pattern of being rude.

(Manager, Consulting)

The next quote is an example from another interviewee who seemed fairly certain initially that she had never received a rude email at work, yet once she got more comfortable, and the question was rephrased to focus on her prior jobs, she easily recalled a rude incident:

In terms of rude emails ... I'm trying to think ... I don't think I've really had one. Sometimes, I am copied on the email, so I wasn't necessarily the person they were responding to directly.

(after question was rephrased, later in the interview)

I remember there were a couple of the guys who worked in sales, and for whatever reason they didn't like the way that I operated, or whatever and I remember I just got this, like, barrage of rude responses and I remember reading the email and I was showing it around the office going I can't believe this man is so rude to me! Like how dare he talk to me in that kind of tone. ... I was just amazed, it was just so completely blatantly rude! (Consultant, Public Relations)

Similarly, the next interviewee initially said she was not bothered by the types of rude behavior encountered during the course of her job, but once the question was approached from a different perspective, and she was asked to describe specific instances of rudeness that she had encountered in the past, it was clear that sometimes incivility was an issue:

I'm trying to even think of ... some people might define the emails that I get as rude but I don't think they are because one, I have a relationship with them (referring to her clients) and I understand how they work and how they communicate. Two, I don't take it personally because I understand the culture I' $m$ in.

(after question was rephrased, referring to a specific incident far in the past) 
... we just kept going back and forth and it was so heated that I even turned to my colleagues to help me respond to this email and just step away and be done with it. Plus, I know the nature of this person, I know how he is, I know how he communicates and I know what he's trying to get so I was feeding into a fire. That was my mistake. I had to stop and get reinforcement to help me level my emotions before responding. I've had incidents with him before and he is just a rude person.

(Program Director, Education)

Next, there was some limited evidence to suggest that employees may conceptualize and discuss rude email in terms that are potentially different than face-to-face rudeness. Most interviewees said that rude email was unique in terms of its features, causes, and effects. Specifically, the following examples indicate how rudeness experienced through email may be different than rudeness in a face- to-face encounter:

A face to face interaction doesn't live forever and you don't get to read it and re-read it, but when somebody sends you that email you can read it over and over and over. And you can analyze it and try to figure it out if you want ... you can answer it. You can answer it and try to calm them down. You can answer it and match them. You can ignore it, you can delete, you can do all kinds of things but a verbal exchange isn't there. You can't relive it ... it's gone.

(Administrative Assistant, Education)

If I were to compare the two communication mediums or avenues, I would say that it probably takes a ruder person to be rude in person than it does over email. You know because, like if I ask you a question face to face you're going to answer me, but if I send you an email, you might not get back to me for quite some time or ever at all. (Assistant Program Director, Education)

One thing which makes a big difference is that if I say something rude to you (in-person), you will likely respond in some way indicating that you are ticked off even more or tempered down, but in the email you're just going to get it all out and the other person has no chance of responding until they read the whole thing. (Software Engineer, Information Technology)

The difference is that I get to tell my manager ... and everyone is fully aware of the situation and you can handle it. But, you get blindsided by an email from somebody that's mad, and you had no idea they were mad. And it's way easier to do because people don't even have to ask to talk to your supervisor ... they just hit that cc button like crazy!

(Administrative Assistant, Education)

Interviewees also indicated a preference for one medium over the other. For instance, some interviewees felt they could be more calculated and rational when handling a contentious situation over email, whereas others felt their ability to express their emotions in-person could soften a message that might be taken the wrong way in email. Here are several perspectives:

I prefer handling a sensitive issue by email. Over email I can filter and edit, whereas phone conversations can escalate much more quickly... and there isn't that time and distance to filter and measure and be diplomatic. (Senior Account Manager, eMarketing Firm)

Email can very easily be miscommunicated. What I could be saying can be rude to the reader when I'm really just enforcing a rule. The outcome is what it is, but over email it may be taken as rude, like "How dare you give me the wrong answer? How dare you give me a no when I demand a yes?" And that will keep going ... whereas if it's in person, I can kill them with kindness and they can see the kindness in my voice and my gestures, which will calm their emotions down. In email they can just keep going.

(Program Director, Education)

“I've learned that there's times where I just don't email and it's either going to be a phone conversation with my client or face to face with somebody in my office if it can be because there's just the chance that it may not go, or not be understood or perceived the way that you're anticipating."

(Title Analyst, Oil and Gas)

Some interviewees also discussed the difference between the two communication mediums in terms of their consequences, and provided examples indicating that email rudeness may have costs that are more severe than rude behavior: 
It's much worse to have a difference of opinion over email. It's like ... do you want to get your point out? Or do you want to just unload? Email is much more passive-aggressive. If you want the issue resolved, you have to go face to face.

(Program Coordinator, Graduate Education)

I think more times than not I would say email may have worse consequences than face to face and I think that it's easier to understand what a person is trying to get across when you can see their facial expressions, when you can hear their tone of voice, their inflections in their voice and a lot of times they may even explain things more, go into more detail than maybe what they would in an email.

(Marketing Director, Non-Profit)

Although a wide range of examples of rude behavior were cited, all were instances that the interviewees themselves referred to as rude. Some of the examples of rudeness provided by interviewees may also overlap with related constructs (i.e., bullying, aggression, flaming, and so on). However, this is expected because there is theoretical overlap between many of these constructs and rudeness. Evidence from the interviews also suggested that admitting to being offended by an email is a sensitive topic or one that employees seemed either to be hesitant to recall intentionally or may not even be aware of. A discussion of the implications of these findings is considered in the following section.

\section{Discussion and implications}

The findings of this research, suggest support for the following three aspects of rudeness: (1) it is common and experienced regularly in a wide-variety of professions and organizations; (2) although specific contextual circumstances vary by situation, participants believe it has negative consequences; (3) participants perceive and articulate tangible differences between rude email and rude face-to-face encounters.

\subsection{The prevalence and frequency of rude behavior at work}

Although the frequency with which interviewees reported encountering rudeness was varied, all interviewees did report experiencing some form of rudeness in their jobs. The specific frequency ranged from weekly to only a few times per year, but may have been due to the lack of consistency from interviewees about what types of behavior constitute rude behavior. For instance, interviewees who reported that they experienced it infrequently, tended to cite more hostile experiences of workplace aggression than the low-intensity types of behavior that are actually representative of rudeness. Employee perceptions of incivility were broader and included more types of workplace mistreatment (i.e., bullying, flaming, and abusive supervision) than what is currently reflected in construct boundaries. In other words, when asked to reflect on instances when they were treated "rudely," without providing a definition of rude behavior or giving examples of rudeness, employees provided a wide range of conceptualizations, from very harsh abusive situations to minor hassles and inconveniences. This inconsistency in the everyday meaning of "rudeness" may help explain the wide range in frequency with which employees reported experiencing uncivil treatment.

Another finding from the study was that discussing personal experiences with workplace rudeness may be more complicated than previously believed. Specifically, talking about their individual involvement with rude incidents was a topic that some employees were hesitant to divulge, unable to fully remember, or potentially one that they had not considered prior to the interview. One reason for this could be that admitting to being offended or hurt by someone may take introspection and a willingness to be vulnerable, two things are not generally acceptable in most business environments. For instance, some interviewees were more forthcoming about their experiences than others. In multiple instances, interviewees initially said, "I just don't get upset about email that others might think of as rude" or "I am not the type of person to take things like that personally." In fact, most interviewees said some variant of this initially. Then, as the issue was discussed further, they seemed to become more comfortable and willing to share their experiences. 
Despite their initial hesitation or reluctance, most interviewees did eventually open up and provided multiple specific instances when they were involved in a situation that they felt was particularly offensive and explained why. Some even shared instances when they had unintentionally acted rudely toward someone else. A few interviewees were even willing to share physical examples of rude emails they had received and kept. Despite some initial hesitation to discuss the topic, evidence from the interviews supports the initial assumption that incivility, either in its electronic form or face-to-face version, does exist in a wide variety of industries, organizations, and jobs.

Future studies should explore employees' reluctance to discuss being offended by rudeness. Is there something about being the target of rudeness on the receiving end that makes people uncomfortable? Why do employees seem to gravitate toward discussing it in the third-person, in the past, or in hypothetical scenarios rather than recalling recent first-person experiences? Does this inclination vary by culture, organization, or position within the company? Is there a time when employees feel more comfortable discussing workplace rudeness? What happens when rude incidents are brought up to other coworkers or a supervisor? An investigation of questions such as these could lead to a fruitful exploration of the interplay between culture and perceptions of rudeness. A valuable contribution for future studies would be to look at how norms, values, and beliefs affect the interpretation of workplace communication and the acceptability of feeling offended at work.

\subsection{Adverse consequences of incivility}

Along with the existence of rudeness, the interview data provides some support for the notion that rude behavior is likely associated with negative consequences for employees. There are several examples that suggest that targets of rudeness experience negative emotions, strained relationships, and a loss of productivity. For example, the most commonly perceived outcome of rudeness was the negative psychological and emotional consequences it generated for employees themselves. The specific types cited in the interviews comprised a wide-range of affective states from dread and anxiety to sadness and shame. For example, several people reported experiencing increased levels of stress and worry, regret, surprise, aggravation, and embarrassment. However, a few employees recalled feeling deeper or more intense emotions such as humiliation, shame, and anger. Although the degree of the specific emotional response likely varied depending on the circumstance, it became clear that exposure to rudeness in an organizational setting was commonly followed by a negative emotional reaction.

Second, interviewees described how incivility negatively influenced their workplace relationships, including their connection with coworkers, clients, and supervisors. Multiple people described tension between their fellow employees and coworkers as a result of a rude encounter. Beyond this, there was also some indication that the interpersonal ramifications of rudeness run deep and are remembered and easily recalled years later when they involve workplace friends. Interviewees reported avoidance, dodging, and escaping as some of the more common ways of dealing with a rude face-to-face encounter. For instance, several interviewees discussed making a conscious and concerted effort to minimize contact with coworkers after being treated rudely. This practice seemed to occur regardless of its impact on the work itself. Therefore, when experienced in a work setting, employees perceived many negative effects of incivility.

Third, most interviewees discussed dealing with rudeness as a very time and labor-intensive process, and one that involved significant attention, taking their focus away from productivity. Specifically, some interviewees recalled spending several hours or more on the construction of a reply to a rude email. In more extreme circumstances, when emotions were very heated, targets of rudeness remembered talking extensively to coworkers about the incident. The anxiety, stress, and lost time spent dealing with the situation seemed to be compounded because other co-workers were involved and also spending their time trying to help the target cope with the situation. Finally, several interviewees reported a tendency to dwell on and ruminate about these experiences, which represents an additional threat to employees' productivity and work quality. In sum, the stories and examples provided here strongly suggest that employees perceive workplace incivility to have a multitude of adverse emotional, relational, and productivity-related consequences.

\subsection{Differences between face-to-face and email rudeness}


A third finding from the qualitative interview data was some initial evidence indicating that email rudeness may be conceptually distinct from face-to-face rudeness. When asked about both types of rudeness, interviewees tended to discuss them separately. For instance, some interviewees brought up distinct features, such as using the carbon-copy (cc) function or not responding to an email as a particularly common way of angering or offending someone. On the contrary, when asked about face-to-face rudeness, interviewees referenced raised voices or expressions as examples of face-to- face rudeness. The different vocabulary and separate examples people used to describe each type of rudeness, indicated some preliminary evidence that they may be conceptualized as two distinct entities with different causes and potentially different consequences.

Similarly, when asked about both types of rudeness directly in comparison, interviewees were able to easily explain nuanced differences. For instance, some interviewees discussed how email rudeness is much more easily ignored, whereas others referenced how the blind-carbon-copy function can be used to gossip and spread rumors. Interviewees also explained how emoticons could be used to both help clarify or diffuse a sensitive message, but they could also be used as a way to be passive-aggressive. Another difference brought up was the use of subject lines, which one interviewee explained was the source of a frustrating situation for him because he had a client who used the same one (his name) for every email, which made organizing their conversations and finding something they had discussed in the past virtually impossible. There were multiple examples of situations where a rude incident that occurred through one medium could not have been replicated in the other.

Further, some interviewees also expressed a preference for dealing with or encountering rudeness through one medium over the other. For example, some employees reported that they would rather handle contentious matters over email because they have more time to think, filter, edit, and respond in a diplomatic and professional manner. In comparison, several others discussed how they believed the outcome was much better when sensitive subjects were talked about in-person. The reasoning behind their preference had to do with the ability to soften a message with smiles, body language, and tone of voice. In addition, some people felt that by using face-to-face communication they could provide more explanation to whomever they were communicating with, and have more certainty that the message was being interpreted as intended. Taken together, these preliminary findings indicate that making a theoretical and empirical distinction between email rudeness and face-to-face rudeness may be an important question for future research to continue to explore.

In sum, the findings of this study suggest initial support for the following three aspects of rudeness. First, it is common and experienced regularly in a wide-variety of industries, professions, and organizations. Second, although the specific contextual circumstances vary by situation, participants believe that it has negative consequences for their attitude, working relationships, and productivity. Finally, participants articulated tangible differences between rude email and rude face-to-face encounters. Most research to date has focused on examining incivility through a quantitative lens, which is important but insufficient for understanding the subtleties of such a complex and multifaceted phenomenon. Taken together, although this research may raise more questions than it answers, it contributes to our current understanding of incivility by complementing existing quantitative findings with the insights gained from interviews that examine employees' actual experiences with rude workplace behavior. 


\section{References}

Andersson, L., and C. Pearson. 1999. Tit for tat? The spiraling effect of incivility in the workplace. Academy of Management Review 24:452-471.

Burgoon, J. K., and D. B. Buller. 1994. Interpersonal deception: III. Effects of deceit on perceived communication and nonverbal behavior dynamics. Journal of Nonverbal Behavior 18:155-184.

Cameron, A. F., and J. Webster. 2011. Relational outcomes of multicommunicating: Integrating incivility and social exchange perspectives. Organization Science 22:754-771. doi:10.1287/orsc.1100.0540.

Cortina, L. M. 2008. Unseen injustice: Incivility as modern day discrimination in organizations. Academy of Management Review 33:5575. doi:10.5465/AMR.2008.27745097.

Cortina, L. M., V. J. Magley, J. H. Williams, and R. D. Langhout. 2001. Incivility in the workplace: Incidence and impact. Journal of Occupational Health Psychology 6:64-80. doi:10.1037/1076-8998.6.1.64.

Deitch, E. A., A. Barsky, R. M. Butz, A. P. Brief, S. Chan, and J. C. Bradley. 2003. Subtle yet significant: The existence and impact of everyday racial discrimination in the workplace. Human Relations 56:1299-1324. doi:10.1177/ 00187267035611002.

Derks, D., A. Bos, and J. Von Grumbkow. 2007. Emoticons and social interaction and the internet: The importance of social context. Computers in Human Behavior 23:842-849. doi:10.1016/j.chb.2004.11.013.

Derks, D., A. Fischer, and A. Bos. 2008. The role of emotion in computer mediated communication: A review. Computers in Human Behavior 24:766-785.

Francis, L., C. Holmvall, and L. O'Brien. 2015. The influence of workload and civility of treatment on the perpetration of email incivility. Computers in Human Behavior 46:191-201. doi:10.1016/j.chb.2014.12.044.

Giacalone, R. A., C. A. Riordan, and P. A. Rosenfeld. 1997. Organizational sabotage: Toward understanding its causes and preventing its recurrence. In Anti-Social Behavior in Organizations, eds. R. A. Giacalone, and J. Greenberg. Newbury Park, CA: Sage Publications.

Giumetti, G. W., A. L. Hatfield, J. L. Scisco, A. N. Schroeder, E. R. Muth, and R. M. Kowalski. 2013. What a rude email! Examining the differential effects of incivility versus support on mood, energy, engagement, and performance in an online context. Journal of Occupational Health Psychology 18:297-309. doi:10.1037/a0032851.

Griffin, R. W., A. O’Leary-Kelly, and J. Collins. 1998. Dysfunctional work behavior in organizations. In Trends in Organizational Behavior, eds. C. L. Cooper, and D. M. Rousseau, Vol. 5, Jai Pr.

Hershcovis, M. S. 2011. "Incivility, social undermining, bullying... oh my!": A call to reconcile constructs within workplace aggression research. Journal of Organizational Behavior 32:499-519. doi:10.1002/job.689.

Ivancevich, J. M. 1986. Life events and hassles as predictors of health symptoms, job performance, and absenteeism. Journal of Occupational Behavior 7:39-51. doi:10.1002/job.4030070106.

Jessmer, S., and D. Anderson. 2001. The effects of politeness and grammar on user perceptions in electronic mail. North American Journal of Psychology 3:331-346.

Johnson, P., and J. Indvik. 2001. Slings and arrows of rudeness: Incivility in the workplace. Journal of Management Development 20:705714. doi:10.1108/EUM0000000005829.

Kato, S., Y. Kato, and D. Scott. 2009. Relationships between emotional states and emoticons in mobile phone email communication in Japan. International Journal on E-Learning 8:385-401.

Lim, V. K. G., and T. S. H. Teo. 2009. Mind your e-manners: Impact of cyber incivility on employees' work attitude and behavior. Information and Management 46:419-425. doi:10.1016/j.im.2009.06.006.

Minor, K. N., I. H. Settles, J. S. Pratt-Hyatt, and C. Brady. 2012. Experiencing incivility in organizations: The buffering effects of emotional and organizational support. Journal of Applied Social Psychology 42:340-372. doi:10.1111/ j.1559-1816.2011.00891.x.

Miron-Spektor, E., D. Efrat-Treister, A. Rafaeli, and O. Schwarz-Cohen. 2011. Others' anger makes people work harder not smarter: The effect of observing anger and sarcasm on creative and analytic thinking. Journal of Applied Psychology 96:1065-1075. doi:10.1037/a0023593.

Naquin, C., L. Belkin, and T. Kurtzberg. 2010. The finer points of lying online: Email versus pen and paper. Journal of Applied Psychology 95:387-394. doi:10.1037/a0018627.

O’Leary-Kelly, A. M., R. W. Griffin, and D. J. Glew. 1996. Organization-motivated aggression: A research framework. Academy of Management Review 21:225-253.

Pearson, C. M., and C. L. Porath. 2005. On the nature, consequences, and remedies of workplace incivility: No time for "nice"? Think again. Academy of Management Executive 19:7-18.

Pearson, C. M., L. M. Andersson, and J. W. Wegner. 2001. When workers flout convention: A study of workplace incivility. Human Relations 54:1387-1419. doi:10.1177/00187267015411001

Penconek, T. 2015. Perspectives on bullying: A qualitative study into academic incivility among nursing students. Alta RN 71:928.

Porath, C. L., and A. Erez. 2007. Does rudeness really matter? The effects of rudeness on task performance and helpfulness. Academy of Management Journal 50:1181-1197. doi:10.2307/20159919.

Porath, C. L., and C. Pearson. 2010. The cost of bad behavior. Organizational Dynamics 39:64-71. doi:10.1016/j. orgdyn.2009.10.006. 
Riordan, M. A., and R. J. Kreuz. 2010. A survey of emotion encoding and interpretation in computer-mediated communication: Reasons for channel choice. Computers in Human Behavior 26:1667-1673.

Taylor, S., A. Bedeian, and D. Kluemper. 2012. Linking workplace incivility to citizenship performance: The combined effects of affective commitment and conscientiousness. Journal of Organizational Behavior 33:878-893. doi:10.1002/job.773.

Turnage, A. K. 2008. Email flaming behaviors and organizational conflict. Journal of Computer-Mediated Communication 13:43-59. doi:10.1111/j.1083-6101.2007.00385.x.

Walther, J., J. Anderson, and D. Park. 1994. Interpersonal effects in computer-mediated interaction: A meta-analysis of social and anti-social communication. Communication Research 21:460-487.

Weatherbee, T. 2010. Counterproductive use of technology at work: Information \& communications technologies and cyberdeviancy. Human Resource Management Review 20:35-44. doi:10.1016/j.hrmr.2009.03.012. 